\title{
Abundancia estacional y distribución espacial de Mysidacea en el Atlántico sudoccidental, estuario de Bahía Blanca $\left(38^{\circ} 42^{\prime}-39^{\circ} 26^{\prime} \mathrm{S}\right.$ y $\left.62^{\circ} 28^{\prime}-61^{\circ} 40^{\prime} \mathrm{W}\right)$
}

\author{
Seasonal abundance and spatial distribution of Mysidacea in Southern West Atlantic, Bahía Blanca \\ Estuary (38 $42^{\prime}-39^{\circ} 26^{\prime} \mathrm{S}$ and $\left.62^{\circ} 28^{\prime}-61^{\circ} 40^{\prime} \mathrm{W}\right)$ \\ Natalia V. Cardelli ${ }^{1}$, Patricia M. Cervellini ${ }^{1}$ y María C. Piccolo ${ }^{2}$ \\ ${ }^{1}$ Departamento de Biología, Bioquímica y Farmacia, Universidad Nacional del Sur, San Juan 670, (8000) Bahía Blanca, Argentina \\ ${ }^{2}$ Instituto Argentino de Oceanografía, C.C. 804, Camino la Carrindanga Km 7,(8000) Bahía Blanca, Argentina \\ nataliacardelli@yahoo.com
}

\begin{abstract}
Two mysids species were present in the Bahia Blanca estuary: Arthromysis magellanica (Cunningham) and Neomysis americana (Smith). They were present in the estuary from August to March. The abundance of A. magellanica was highest, 31.92 ind. $\mathrm{m}^{-3}$ in spring and $N$. americana abundance reached a maximum of 10.91 ind. $\mathrm{m}^{-3}$ during midsummer. These high abundance values were coincident with the increment of the water temperature. The mean temperature value registered during the sampled period was $15.7^{\circ} \mathrm{C}$. Both species were present from $10^{\circ} \mathrm{C}$ to $23.3^{\circ} \mathrm{C}$ and were absent from $6.6^{\circ} \mathrm{C}$ to $22^{\circ} \mathrm{C}$.

In agreement with the spatial distribution of both species, a relationship in the abundance of $A$. magellanica for the three sampling stations was not detected (ANOVA, $n=8, P>0.80$ ) but there was a relationship in the abundance of $N$. americana for the three sampling stations (ANOVA, $n=10, P<0.05$ ) since the highest density of this species was recorded in the inner zone of the estuary.

Significant differences in salinity were found between the three sampling stations for those dates when $N$. americana was present (ANOVA, $\mathrm{n}=10, P<0.05$ ) but it could not be demonstrated that this variable is related with the spatial distribution of this species in the estuary (covariable, $P>0.15$ ).
\end{abstract}

Key words: Crustacea, species, number of individuals, distribution, Argentina
Resumen.- Dos especies de misidáceos estuvieron presentes en el estuario de Bahía Blanca: Arthromysis magellanica (Cunningham) y Neomysis americana (Smith). Durante el periodo de estudio ambas especies fueron detectadas desde agosto hasta marzo. A. magellanica fue más abundante durante la primavera, con un valor de 31,92 ind. $\mathrm{m}^{-3}$ mientras que $N$. americana lo fue durante el verano, con un valor de 10,91 ind. $\mathrm{m}^{-3}$. Estos altos valores de abundancia fueron coincidentes con el aumento de la temperatura del agua. La temperatura media registrada durante el período de muestreo fue de $15,7^{\circ} \mathrm{C}$. El rango de temperatura de presencia para ambas especies fue de $10^{\circ} \mathrm{C}$ a $23,3^{\circ} \mathrm{C}$. El rango de temperatura en el cual ambas especies estuvieron ausentes fue de $6,6^{\circ} \mathrm{C}$ a $22^{\circ} \mathrm{C}$.

En cuanto a la distribución espacial, no se detectó una relación en la abundancia de $A$. magellanica para las tres estaciones de muestreo (ANOVA, $\mathrm{n}=8, P>0,80$ ). Sí existió una relación en cuanto a la abundancia de $N$. americana (ANOVA, $\mathrm{n}=10$, $P<0,05)$, ya que de esta especie se capturó un mayor número de individuos en la zona más interna del estuario.

Se hallaron diferencias significativas en relación con la salinidad entre las tres estaciones de muestreo para aquellas fechas en la que $N$. americana estuvo presente (ANOVA, $\mathrm{n}=10, P<0,05$ ), pero no se pudo demostrar que esta variable estuviera relacionada con la distribución espacial de esta especie en el estuario (covariable, $P>0,15$ ).

Palabras clave: Crustacea, especies, número de individuos, distribución, Argentina 


\section{Introducción}

Los misidáceos son un grupo de crustáceos que poseen posibilidades de colonizar diferentes ambientes ya sean continentales, marinos o estuarinos. En el mar se los encuentra en la columna de agua, sobre o por encima del fondo, en cuevas y como comensales de otros animales. La mayoría de los misidáceos costeros realiza una migración vertical, ascendiendo y dispersándose en la columna de agua durante la noche y descendiendo hacia mayor profundidad durante el día (Mauchline 1980, Murano 1999). También existen datos de migraciones dentro y fuera de los estuarios; estos movimientos entre el estuario propiamente dicho y zonas costeras aledañas podrían sugerir patrones estacionales y responder a variaciones en las condiciones ambientales (Hesthagen 1973).

Los misidáceos son filtradores omnívoros, consumiendo detritos orgánicos, cuerpos y apéndices de pequeños crustáceos y diatomeas (Kaester 1970, Murano 1999). También se han observado hábitos de carnivoría en algunas especies, alimentándose de copépodos, cladóceros, pequeños moluscos, anfípodos y aún de otros misidáceos.

Los misidáceos constituyen un componente importante en la biomasa del zooplancton de los estuarios y de las regiones costeras (Jerling \& Wooldridge 1995). La alta biomasa alcanzada por estas poblaciones en esos ecosistemas sugiere que ellos pueden jugar un rol en la cadena alimenticia, en particular como fuente de alimento de diferentes especies de peces que usan esos ambientes como "nurseries" o zonas de cría (Brown \& Mclachlan 1990, Chigbu \& Sibley 1994, Redon et al. 1994, Gibran \& Castro 1999, Morte et al. 1999).

La fauna de misidáceos en aguas del Atlántico sudoccidental es pobremente conocida; Ramírez (1976) halló que la especie Arthromysis magellanica (Cunningham) constituye, en los meses de verano, el principal rubro alimenticio de Sprattus fueguensis (Jenyns) (sardina fueguina) en las costas de Santa Cruz (4945’S), Argentina. En nuestra zona, López Cazorla (1987) señala la importancia de las dos únicas especies de misidáceos Arthromysis magellanica y Neomysis americana (Smith) en la dieta de varias especies de peces presentes en el estuario de Bahía Blanca tales como la corvina (Micropogonias furnieri (Desmarest), el lenguado Paralichtys orbignyanus (Jenyns), la raya
(Synpterygia spp.) y el pejerrey (Odonthesthes spp.), llegando a la misma conclusión sobre la alimentación de la pescadilla de red Cynoscion striatus (Cuvier) (López Cazorla 1996).

Los conocimientos sobre la fauna de misidáceos en nuestra área de estudio son escasos, particularmente están basados en caracteres morfológicos y taxonómicos (Guerrero et. al. 1976, Ferrarello 1988, Hoffmeyer 1983, 1990) es por este motivo que se analizaron las poblaciones de misidáceos a lo largo de un ciclo anual en el estuario de Bahía Blanca. Los objetivos propuestos fueron identificar las especies presentes, describir su distribución espacial, analizar los cambios estacionales en la abundancia numérica y relacionar esos cambios con variables ambientales.

\section{Materiales y métodos}

El estuario de Bahía Blanca se encuentra ubicado en la costa atlántica de la provincia de Buenos Aires, Argentina, entre los $38^{\circ} 42^{\prime}$ y $39^{\circ} 26^{\prime} \mathrm{S}$ y $62^{\circ} 28^{\prime}$ y $61^{\circ} 40^{\prime} \mathrm{W}$ y presenta una longitud aproximada de 100 $\mathrm{km}$. Se halla formado por una serie de canales orientados en dirección NW-SE y extensas planicies de mareas e islas, contando con una superficie total de $2.290 \mathrm{~km}^{2}$ (Perillo \& Piccolo 1987). Tiene forma clásica de embudo, con una escasa influencia de agua dulce, altas velocidades de corriente, mezcla turbulenta y la amplitud de marea decrece hacia la zona externa de la bahía. (Fig. 1).

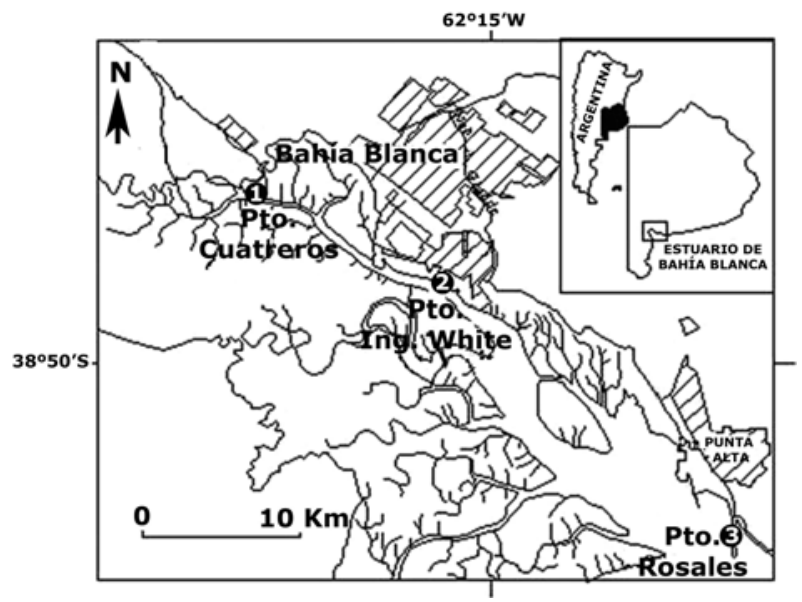

Figura 1

Ubicación de las estaciones de muestreo 1-3, en el estuario de Bahía Blanca

Location of sampling stations 1-3 at Bahía Blanca estuary 
Debido a las diferencias existentes en cuanto a su física, su química y a su biología, este estuario puede ser dividido en dos zonas: una interna donde las características abióticas son marcadamente fluctuantes (entre la localidad de Villarino y Puerto Ingeniero White) y otra externa donde las condiciones ambientales se hacen mucho más estables acercándose a las del ambiente marino (desde Puerto Ingeniero White, hasta una línea imaginaria que une Punta Laberinto y Faro Monte Hermoso) (Mianzan \& Sabatini 1985).

Basándonos en la distribución de salinidad y temperatura el estuario de Bahía Blanca muestra un patrón verticalmente homogéneo (Perillo et al. 2004).

La temperatura media anual del agua superficial es de $13^{\circ} \mathrm{C}$ variando entre $21,6^{\circ} \mathrm{C}$ en verano a $8,5^{\circ} \mathrm{C}$ en invierno (Perillo \& Piccolo 1987). En la región interna los valores de salinidad fluctúan ampliamente entre 17 y 41 ups y en la región más externa se hallan salinidades semejantes a las de la plataforma continental (Piccolo \& Perillo 1997).

Las muestras diurnas fueron obtenidas quincenalmente en tres estaciones de muestreo, localizadas en estrecha relación con la zonación que presenta el estuario de Bahía Blanca. El periodo de estudio abarcó desde el 22 de febrero de 2002 hasta el 12 de marzo de 2003. (Estación 1: Puerto Cuatreros y Estación 2: Puerto Ingeniero White, representativa de la zona interna y Estación 3: Puerto Rosales, zona externa).

El muestreo se realizó desde el muelle por medio de 20 lances verticales desde $4 \mathrm{~m}$ de profundidad hasta la superficie con una red cónica de $30 \mathrm{~cm}$ de diámetro y $200 \mu \mathrm{m}$ de abertura de poro de malla, realizándose los muestreos siempre en el mismo estado de marea (subiente). El volumen de agua filtrado fue estimado utilizando el radio de la boca de la red, la profundidad y el número de lances. Se efectuaron lances repetidos, hasta obtener un volumen representativo $\left(8 \mathrm{~m}^{3}\right.$ por estación de muestreo). Los valores de temperatura y salinidad del agua fueron determinados en cada estación de muestreo. La temperatura del agua de mar se midió con termómetro de superficie y la salinidad fue analizada en el laboratorio de Química Marina del Instituto Argentino de Oceanografía, usando un salinómetro Horiba Water Quality U-10. El zooplancton fue conservado en una solución de formalina al 4\%, los individuos fueron luego identificados al nivel de especie siguiendo a Tattersall (1955), Brunel (1960), González (1974), Ferrarello (1988), Hoffmeyer (1990), Tavares \& Bond-Buckup (1990), Crescenti (1997) y Murano (1999). Para el conteo se utilizó una lupa binocular Olympus con ocular micrométrico y los valores de abundancias fueron expresados como el número de individuos por metro cúbico de agua (ind. $\mathrm{m}^{-3}$ ).

Para la variable abundancia (ind. $\mathrm{m}^{-3}$ ) se utilizó la clásica transformación logarítmica a los datos como sigue: Ln (ind. $\mathrm{m}^{-3}+1$ ) y se consideró un ANOVA doble (estaciones por fecha). Las comparaciones de a pares entre las estaciones de muestreo, se realizaron con diferencia mínima significativa de Fisher (DMS). Para ello se utilizaron las fechas con datos completos y que tuvieran un promedio mayor que cero. Los promedios estimados fueron llevados a la escala original (ind. $\mathrm{m}^{-3}$ ) por retransformación. Como existió una obvia diferencia estacional de abundancia en ambas especies (hay varias fechas con promedio cero), para relacionarla con la temperatura, se aplicó el coeficiente de correlación biserial-puntual. Primero se dividió en dos la variable abundancia (presencia=1, ausencia=0) en cada fecha. Luego se aplicó el coeficiente de correlación habitual entre la variable continua (temperatura media de la fecha) y la binaria. La expresión resultante se relaciona directamente con la diferencia entre el promedio de temperaturas de las fechas en que la especie estuvo presente, versus el promedio de temperaturas de las fechas en que estaba ausente. Como existieron diferencias en los promedios de abundancia entre las estaciones de muestreo, la variable salinidad se agregó como covariable al ANOVA original.

\section{Resultados}

Se identificaron dos especies de misidáceos en el estuario de Bahía Blanca: Neomysis americana y Arthromysis magellanica. De los datos obtenidos se aprecia que durante los meses de primavera y comienzos del verano, la especie predominante fue $A$. magellanica mientras que finalizando el período estival lo fue $N$. americana, aunque con valores medios de abundancia muy por debajo de lo observado para $A$. magellanica (Fig. 2). La aparición de individuos de ambas especies se produjo a fines de agosto de 2002 y continuó hasta fines de marzo de 2003. 


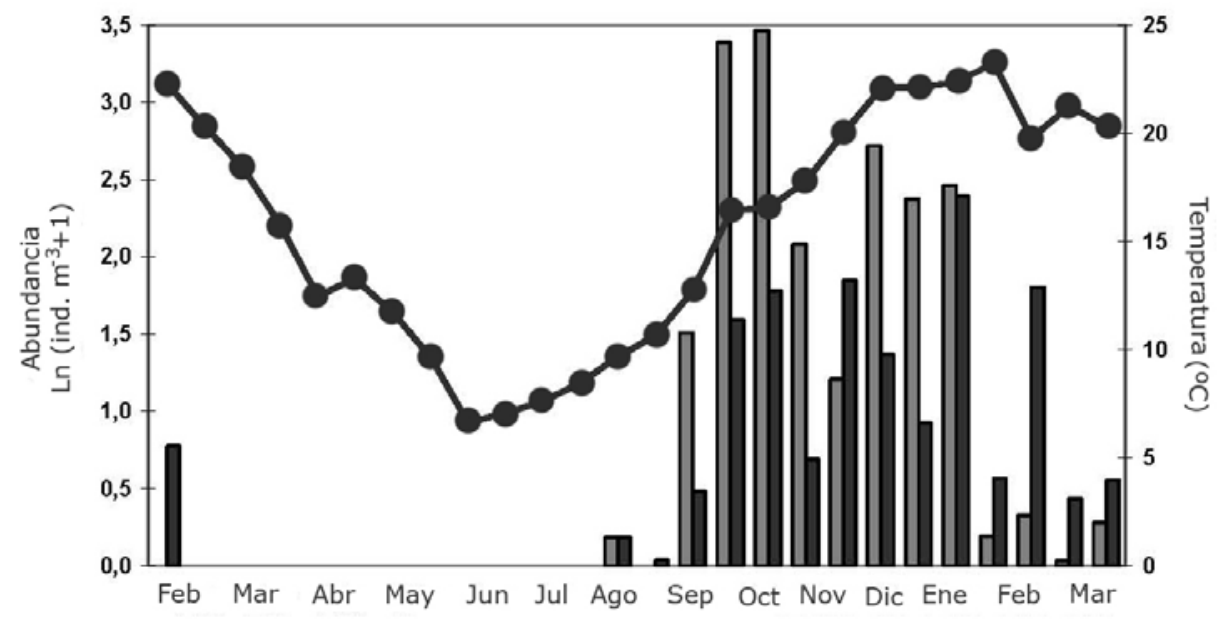

Figura 2

\section{Abundancia media de Arthromysis magellanica (barras grises) y Neomysis americana (barras negras) en relación con la temperatura para cada fecha de muestreo}

Mean abundance of Arthromysis magellanica (grey bars) and Neomysis americana (black bars) related with temperature for each sampling date

Durante el periodo de estudio fueron observados para la especie A. magellanica valores de abundancia que fluctuaron entre un mínimo de 1,03 ind. ${ }^{-3}$ en marzo de 2003 y un máximo de 31,92 ind. $\mathrm{m}^{-3}$ en octubre de 2002. N. americana presentó un mínimo valor de abundancia de 1,03 ind. $\mathrm{m}^{-3}$ en septiembre de 2002 y una densidad máxima de 10,91 ind. $\mathrm{m}^{-3}$ en enero de 2003.

Durante el periodo de estudio la temperatura y la salinidad evidenciaron un patrón verticalmente homogéneo en la columna de agua, por lo tanto los datos superficiales fueron utilizados como representativos de toda la columna.

La abundancia para ambas especies evidenció una marcada variación estacional y la temperatura del agua acompañó dicha variación. Existió una correlación altamente significativa entre la temperatura y presenciaausencia de cada especie (A. magellanica $\mathrm{r}=0,57 ; N$. americana $\mathrm{r}=0,60)$. La temperatura media registrada durante el período de muestreo fue de $15,7^{\circ} \mathrm{C}$. El rango de temperatura de presencia para ambas especies fue de $10^{\circ} \mathrm{C}$ a $23,3^{\circ} \mathrm{C}$, con una temperatura promedio de $18,8^{\circ} \mathrm{C}$ para la especie A. magellanica y de $18,5^{\circ} \mathrm{C}$ para $N$. americana. El rango de temperatura en el cual ambas especies estuvieron ausentes fue de $6,6^{\circ} \mathrm{C}$ a $22^{\circ} \mathrm{C}$, con un promedio de $12,6^{\circ} \mathrm{C}$ y $11,9^{\circ} \mathrm{C}$ respectivamente.
Para estudiar la distribución espacial de las especies presentes en el estuario se analizaron estadísticamente sus abundancias a lo largo de las tres estaciones muestreadas (Fig. 3). Para A. magellanica no se hallaron diferencias en abundancia entre las tres estaciones de muestreo (ANOVA, $\mathrm{n}=8, P>0,80$ ) pero sí se detectaron diferencias significativas para $N$. americana (ANOVA, $\mathrm{n}=10, P<0,05)$. El valor máximo de abundancia de esta última especie se registró en la estación más interna (Puerto Cuatreros) y el mínimo valor en la estación más externa del estuario (Puerto Rosales).

Ambas especies estuvieron presentes en un rango de salinidad que fluctuó entre 17,23 y 34,01 ups. Como ya se mencionó anteriormente, al detectarse diferencias significativas en cuanto a la distribución espacial de la especie $N$. americana, se propuso relacionar la abundancia de dicha especie con la variable salinidad, para reconocer si esta variable podría afectar su distribución en el estuario. Si bien los valores medios de abundancia de esta especie se relacionaron inversamente con las medias respectivas de salinidad, esta relación no se correspondió fecha a fecha (covariable no significativa, $P>0,15)$. El valor medio de salinidad registrado para la estación más interna (Puerto Cuatreros) fue de 27,953 ups mientras que en la estación más externa (Puerto Rosales) se obtuvo un valor medio de salinidad de 31,361 ups (Fig. 4). 


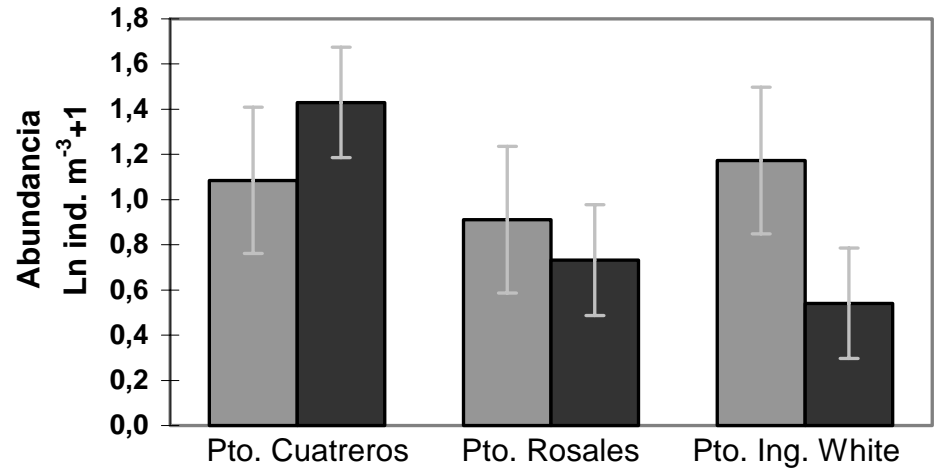

Figura 3

Promedio de abundancia de Arthromysis magellanica (barras grises) y Neomysis americana (barras negras) en cada estación de muestreo

Mean abundance of Arthromysis magellanica (grey bars) and Neomysis americana (black bars) in each sampling station

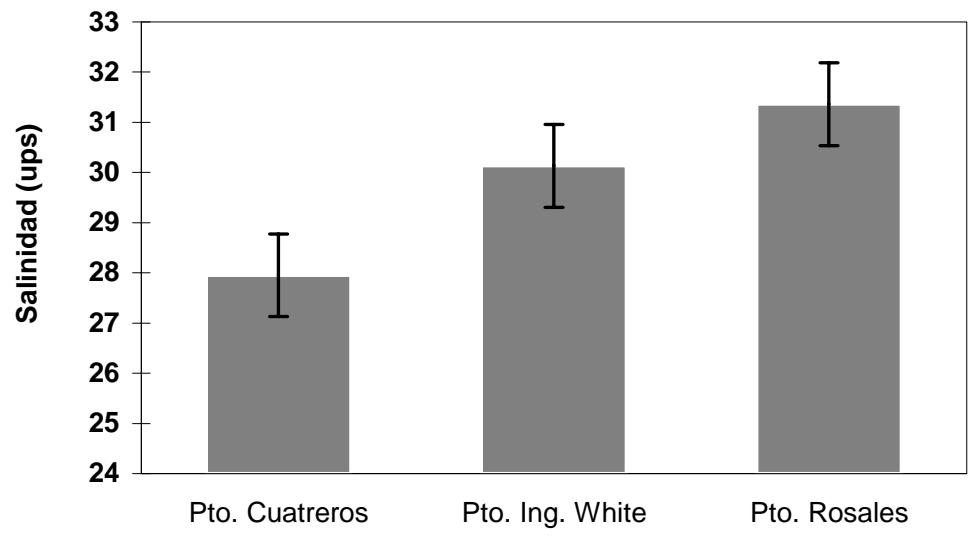

Figura 4

Valores medios de salinidad \pm 1 ES medidos en cada una de las estaciones de muestreo

Mean salinity values \pm 1 SE measured in each sampling station

\section{Discusión}

Según la literatura la especie Neomysis americana se distribuye en la costa este de América del Norte en el golfo de St. Lawrence, Canadá, hasta las costas del estado de Virginia en Estados Unidos. También se la ha detectado en Brasil, Uruguay y en Argentina, en la bahía de San Borombón $\left(30^{\circ} \mathrm{S}-40^{\circ} \mathrm{S}\right)$, el estuario de Bahía Blanca y Bahía Anegada (Provincia de Buenos Aires) (González 1974, Mauchline \& Murano 1977, Hoffmeyer 1990). La distribución que se conoce hasta el momento de Arthromysis magellanica se restringe a Argentina; en particular se la encuentra en el estuario de Bahía Blanca (Provincia de Buenos Aires), Estrecho de Magallanes (Provincia de Santa Cruz, 4945'S) y una 
zona intermedia entre las Islas Malvinas y Tierra del Fuego (45은 $\left.53^{\circ} \mathrm{S}\right)$ (Tattersall 1955, Crescenti 1997).

Los misidáceos son conocidos como un importante componente en comunidades de áreas costeras. Sí bien en ambientes similares al estuario de Bahía Blanca la fauna de estos crustáceos es muy diversificada (Wigley \& Burns 1971, Tavares \& Bond-Buckup 1990, Zouhiri et al. 1998), en nuestra área de estudio solamente fueron registradas dos especies Arthromysis magellanica y Neomysis americana, de esta forma el estuario de Bahía Blanca presentaría una baja riqueza específica con respecto a estos crustáceos.

El período de mayor abundancia para $A$. magellanica se produjo en octubre, con un valor de 31,92 ind. $\mathrm{m}^{-3}$, coincidente con lo observado por Ferrarello (1988) aunque en su trabajo no se indican valores numéricos de abundancia. En la actualidad no se cuenta con datos de abundancia que puedan ser comparados con los resultados hallados en el estuario. Principalmente, esto es debido a que los estudios existentes sobre $A$. magellanica se han basado exclusivamente en su morfología y posición taxonómica.

Con respecto a la etapa de aparición en el plancton, esta especie estuvo presente entre agosto y marzo. Al igual que en nuestro trabajo, Hoffmeyer (1983), muestreando la misma área de estudio, detectó la presencia de ejemplares para los meses de primavera y verano. Por el contrario, Ferrarello (1988), describiendo los diferentes estadios del desarrollo larval de $A$. magellanica en el estuario de Bahía Blanca, halló ejemplares a lo largo de todo el año, aunque en su estudio el autor localizó las estaciones de muestreo en sitios cercanos a la boca del mismo, tales como Bahía Verde, Bahía Falsa y Canal Bermejo.

$N$. americana estuvo presente en el estuario de Bahía Blanca con densidades poblacionales que fluctuaron entre un mínimo (1,03 ind. $\mathrm{m}^{-3}$ ) en septiembre de 2002 y un máximo $\left(10,91\right.$ ind.m $\left.{ }^{-3}\right)$ en enero de 2003, no hallándose ejemplares entre los meses de marzo a julio. Viñas et. al. (2005) en la Bahía de San Borombón registró las máximas densidades en el verano (febrero). Hoffmeyer (1983), estudiando la zona interna y media del estuario de Bahía Blanca (Puerto Cuatreros y Puerto Ingeniero White), detectó para esta segunda especie, ejemplares entre septiembre y mayo, hallando las mayores densidades en septiembre y marzo. Esta abundancia significativa durante ambos meses, concordó con nuestros resultados. Por otra parte, se observaron ciertas diferencias en cuanto al pico de mayor abundancia, el cual fue más significativo durante el verano (enero) y no en la primavera como lo ha señalado Hoffmeyer (1983). También se han observado diferencias a nivel del segundo pico de abundancia, el cual se detectó en nuestro estudio en el mes de noviembre. Esta misma autora continuó hallando escasos ejemplares en los dos meses siguientes a marzo, sin embargo, Viñas et al. (2005), en la Bahía de San Borombón, encontró ejemplares a lo largo de todo el año.

Hulburt (1957) para el estuario de Delaware River encuentra abundancias inferiores a 0,10 ind. $\mathrm{m}^{-3}$ en superficie, mientras que en muestras de fondo concentraciones mayores a los 10 ind. $\mathrm{m}^{-3}$ siendo el máximo valor hallado de 72,46 ind. $\mathrm{m}^{-3}$. Callieri et al. (2001) para el estuario Solis Grande en Uruguay, hallaron densidades poblacionales de $N$. americana mucho más elevadas a las encontradas en este estudio, estos altos valores estuvieron cercanos al máximo valor registrado en la literatura para los misidáceos, (i.e. $10^{3}$ ind. $\mathrm{m}^{-3}$; Zouhiri et al. 1998), en la actualidad no se cuenta con más datos de referencia para esta especie en estuarios de similares características.

Al igual que otros crustáceos de aguas poco profundas, muchas especies de misidáceos migran hacia aguas más profundas en el otoño y regresan en primavera o verano, como Neomysis integer, Mesopodopsis slabberi y Praunus flexuosus (Kaester 1970, Almeida Prado 1973). Este comportamiento de migración podría explicar en el estuario de Bahía Blanca la ausencia de individuos de las dos especies durante el período de otoño-invierno.

Los picos de abundancia para ambas especies, concordaron con lo observado por otros autores para el holoplancton en ambientes similares al estuario de Bahía Blanca. Acartia tonsa es la especie de copépodo dominante en el estuario de Bahía Blanca y fue registrado a lo largo de todo el año; esto también fue señalado por González (1974) para el Río de la Plata y por Hoffmeyer (1994) para el estuario de Bahía Blanca.

Se halló una variabilidad estacional en la presencia de misidáceos que se correspondió con los cambios estacionales de temperatura; es por este motivo que a temperaturas más altas se encontraron mayores abundancias de ambas especies y escasos ejemplares a temperaturas más bajas. Sin embargo, no se puede 
hablar de una relación causal ya que nuestro estudio se basó en un análisis general de los datos de presencia y ausencia de ambas especies. El rango de temperatura en el cual ambas especies estuvieron presentes en el estuario de Bahía Blanca fue coincidente con lo señalado por Ferrarello (1988) para A. magellanica, trabajando en la misma área de estudio, quien la halló entre $8,8^{\circ} \mathrm{C}$ y $22,7^{\circ} \mathrm{C}$ y por Calliari et al. (2001) para $N$. americana en el estuario Solis Grande en Uruguay, quienes la encontraron entre $11,2^{\circ} \mathrm{C}$ y $25^{\circ} \mathrm{C}$.

En cuanto a la distribución espacial, $N$. americana fue más abundante en la zona interna del estuario (Puerto Cuatreros), presentando densidades más bajas en la estación externa (Puerto Rosales). Estas observaciones también han sido señaladas por Hoffmeyer \& Mianzan (2004) para la misma zona de estudio. Las diferencias halladas en cuanto a su distribución en el espacio podrían estar relacionadas al hecho que esta especie soportaría amplias fluctuaciones de las variables ambientales, fenómeno que ocurre en la zona interna del estuario de Bahía Blanca (Mianzan \& Sabatini 1985).

A. magellanica, por el contrario, presentó un comportamiento diferente en cuanto a su distribución espacial; se la halló a lo largo de las tres estaciones de muestreo, lo que evidenciaría una preferencia no marcada en cuanto a su distribución espacial. No obstante los valores de mayor abundancia fueron registrados en la estación más externa (Puerto Rosales). Esta última característica fue señalada también por Hoffmeyer \& Mianzan (2004).

Se encontraron valores de salinidad muy similares a los registrados por otros autores para el estuario de Bahía Blanca (Mianzan \& Sabatini 1985, Piccolo \& Perillo 1997). Ambas especies estuvieron presentes en un rango de salinidad que fluctuó entre 17,23 y 34,01 ups. Hulburt (1957), estudiando la distribución de $N$. americana en el estuario de Delaware River, observó que la isohalina 4 marcaba el límite de entrada de los misidáceos hacia el río, indicando una gran resistencia de esta especie a valores muy bajos de salinidad. Ferrarello (1988), estudiando A. magellanica en la boca del estuario de Bahía Blanca, halló valores máximos de abundancia para esta especie en un rango de salinidad entre 31,5- 33,4 ups. Esto apoyaría lo detectado para nuestra área de estudio ya que se obtuvo la mayor abundancia en la estación más externa (Puerto Rosales), donde el valor medio de salinidad fue de 31,36 ups.
Los valores medios de abundancia de N. americana no se relacionaron con las medias respectivas de salinidad fecha a fecha, es por ello que se concluye que la salinidad no es un factor causal, aunque cuando esta especie está presente en el estuario, tendría preferencia por valores bajos de salinidad. Así, en la estación más externa (Puerto Rosales) donde los valores de salinidad fueron mayores, se observaron valores bajos de abundancia de esta especie y viceversa para la estación más interna (Puerto Cuatreros).

Aunque si bien, la salinidad y la temperatura por sí solas no serían los únicos factores causales de la distribución espacial de estas especies, habría que tener en cuenta además otras características tales como, intensidad de la luz, posibles variaciones en las condiciones ambientales (vientos, velocidad de corriente), entre otros (Mauchline 1980, Calliari et al. 2001).

\section{Agradecimientos}

Los autores expresan su agradecimiento a los integrantes del Laboratorio de Zoología de Invertebrados (Universidad Nacional del Sur), a los integrantes del Instituto Argentino de Oceanografía (IADO) por su asistencia técnica durante el muestreo y a los revisores anónimos por sus valiosos comentarios que permitieron mejorar este trabajo. Este estudio fue llevado a cabo bajo el financiamiento del proyecto PICT $\mathrm{N}^{\circ} 12421$ del Consejo Nacional de Investigaciones Científicas y Técnicas (CONICET) de Argentina.

\section{Literatura citada}

Almeida Prado MS. 1973. Distribution of Mysidacea (Crustacea) in the Cananeia region. Boletin Zoologia e Biologia Marinha 30: 395-417.

Brown AC \& A Mclachlan. 1990. Ecology of sandy shores, 340 pp. AC Brown \& A Mclachlan (eds), Elsevier Science, The Netherlands.

Brunel P. 1960. Artificial key to the Mysidacea of the Canadian Atlantic Continental Shelf. Canadian Journal of Zoology 38: 851-855.

Calliari D, G Cervetto \& M Gómez. 2001. Short-term variability in abundance and vertical distribution of the opossum shrimp Neomysis americana in the Solis Grande River Estuary, Uruguay. Atlántica 23: 117-125. 
Chigbu P \& TH Sibley. 1994. Relationship between abundance, growth, egg size and fecundity in a landlocked population of longfin smelt, Spirinchus thaleichthys. Journal of Fish Biology 45(1): 1-15.

Crescenti N. 1997. Mysids, Systematic account in Amphipods, euphausiids, mysids, ostracods and Chaetognaths. En: Guglielmo L \& A Ianora (eds), Atlas of Marine Zooplankton Straits of Magellan, pp. 133-156. Springer-Verlag, Berlín.

Ferrarello CN. 1988. Descripción morfológica de los estadios de desarrollo de Arthromysis magellanica y algunos aspectos relacionados con su biología. Tesis de Licenciatura en Biología Marina. Universidad Nacional del Sur, Bahía Blanca, Argentina, 44 pp.

Gibran FZ \& RMC Castro. 1999. Activity, feeding behaviour and diet of Ogcocephalus vespertilio in Southern West Atlantic. Journal of Fish Biology 55: 588595.

González LA. 1974. Hallazgo de Neomysis americana en el Río de la Plata. Revista de Biología del Uruguay 2: 119130.

Guerrero MAT, MNC de Izquierdo \& SEP de Canelo. 1976. Observaciones mensuales sobre algunos aspectos biológicos de la Ría de Bahía Blanca, entre Ingeniero White y Puerto Cuatreros. Contribución Científica IADO 36:1-39.

Hesthagen IH. 1973. Diurnal and seasonal variations in the near bottom fauna-the hyperbenthos- in one of the deeper channels of the Kieler Bucht (westwrn Baltic). Kieler Meeresforschungen 29:116-140.

Hoffmeyer MS. 1983. Zooplancton del área interna de la Bahía Blanca (Buenos Aires, Argentina). I. Composición faunística. Historia Natural 3(8): 73-94.

Hoffmeyer MS. 1990. The occurrence of Neomysis americana in two new localities of the south american coast. Crustaceana 58(2): 186-192.

Hoffmeyer MS. 1994. Seasonal succession of Copepoda in the Bahía Blanca estuary. Hydrobiologia 292/293: 303308.

Hoffmeyer M \& H Mianzan. 2004. Macrozooplancton del estuario y aguas costeras adyacentes. En: Piccolo C \& M Hoffmeyer (eds), Ecosistema del estuario de Bahía Blanca, pp.143-151. Editorial Sapienza, Argentina.
Hulburt EM. 1957. The distribution of Neomysis americana in the Estuary of the Delaware River. Limnology and Oceanography 2: 1-11.

Jerling HL \& TH Wooldridge. 1995. Feeding of two mysids species on plankton in temperature South African estuary. Journal of Marine Biology and Ecology 188:243-259.

Kaester A. 1970. Invertebrate Zoology. Crustacea Vol 3, 522 pp. Interscience Publishers, Nueva York.

López Cazorla AC. 1987. Contribución al conocimiento de la ictiofauna marina del área de Bahía Blanca. Tesis Doctoral. Facultad de Ciencias Naturales y Museo. Universidad Nacional de la Plata, Argentina, 247 pp.

Lopez Cazorla AC. 1996. The food of Cynoscion striatus (Cuvier) in the Bahía Blanca area, Argentina. Fisheries Research 28: 371-379.

Mauchline J. 1980. The biology of Mysids and Euphausiids. Advances in Marine Biology 18: 1-681.

Mauchline J \& M Murano. 1977. World list of the Mysidacea, Crustacea. Journal of the Tokyo University of fisheries $\mathrm{V}^{\circ} 4$, 64(1): 39-88.

Mianzan H \& M Sabatini. 1985. Estudio preliminar sobre distribución y abundancia de Muemiopsis macradyi en el estuario de Bahía Blanca (Argentina) (Ctenophora). Spheniscus 1: 53-68.

Morte S, MJ Redon \& A Sanz-Brau. 1999. Feeding habits of Trachinus draco off the eastern coast of Spain. Vie et Milieu 49(4): 287-291.

Murano M. 1999. Mysidacea. En: Boltovskoy D (ed), South Atlantic Zooplankton (1 \& 2), 1706 pp. Backhuys Publs., Leiden, Holanda.

Perillo GME \& MC Piccolo. 1987. Estudio preliminar de la hidrografía y circulación del estuario del río Chubut. Instituto Argentino de Oceanografía - Dirección de protección ambiental, Informe técnico, 59 pp.

Perillo GME, MC Piccolo, ED Palma, DE Pérez \& JO Pierini. 2004. Oceanografía Física. En: Piccolo C \& M Hoffmeyer (eds), Ecosistema del estuario de Bahía Blanca, pp. 61-67. Editorial Sapienza, Argentina.

Piccolo MC \& GME Perillo. 1997. Geomorfología e Hidrografía de los estuarios. En: Boschi EE (ed), El mar Argentino y sus recursos pesqueros. Tomo 1: 133-161. Instituto Nacional De Investigación y Desarrollo Pesquero, Mar del Plata, Argentina. 
Ramírez FC. 1976. Contribución al conocimiento del espectro alimenticio de la sardina fueguina. Neotrópica 22(68): 137-141.

Redon MJ, MS Morte \& A Sanz-Brau. 1994. Feeding habits of the spotted flounder Citharus linguatula off the eastern coast of Spain. Marine Biology 120(2): 197-201.

Tattersall O S. 1955. Mysidacea. Discovery Reports 28: 1190.

Tavares LM \& G Bond-Buckup. 1990. Os Mysidacea da região litorãnea e estuarina de Tramandí, RS, Brasil. Revista Brasileira de Zoologia 7(1-2): 47-57.

Viñas MD, FC Ramírez \& HW Mianzan. 2005. Annual population dynamics of the opossum shrimp Neomysis americana (Smith, 1873) (Crustacea: Mysidacea) from an estuarine sector of the Argentine sea. Scientia Marina 69(4): 493-502.

Wigley RL \& BR Burns. 1971. Distribution and biology of Mysids from the Atlantic coast of the United States in the NMFS Woods Hole Collection. Fishery Bulletin 69(4): 717-746.

Zouhiri S, C Vallet, P Mouny \& J Dauvin. 1998. Spatial distribution and biological rhythms of suprabenthic mysids from the English Channel. Journal of the Marine Biological Association of the United Kingdom 78(4): 1181-1202.

Recibido el 25 de abril de 2006 y aceptado el 18 de agosto de 2006 